\title{
Coexistence and Phase Separation in Sheared Complex Fluids
}

\author{
Peter D. Olmsted ${ }^{1 *}$ and C.-Y. D. $\mathrm{Lu}^{2 \dagger}$ \\ ${ }^{1}$ Department of Physics, University of Leeds, Leeds LS2 9JT, UK and ${ }^{2}$ Polymer and Colloids, Cavendish Laboratory, \\ Cambridge University, Madingley Road, Cambridge CB3 OHE, UK
}

(June 17, 2021)

\begin{abstract}
We demonstrate how to construct dynamic phase diagrams for complex fluids that undergo transitions under flow, in which the conserved composition variable and the broken-symmetry order parameter (nematic, smectic, crystalline, etc. ) are coupled to shear rate. Our construction relies on a selection criterion, the existence of a steady interface connecting two stable homogeneous states. We use the (generalized) Doi model of lyotropic nematic liquid crystals as a model system, but the method can be easily applied to other systems, provided non-local effects are included.
\end{abstract}

Complex fluids in shear flow display a range of behaviors which is only beginning to be unearthed [1 6 . Shear can perturb equilibrium phase transitions (e.g. the isotropic-to-nematic (I-N) liquid crystalline [2,7 9] and isotropic-to-lamellar [10] transitions), and induce structures, (e.g. bilayer onions [4]) that exist only as metastable equilibrium phases. A related phenomenon is dynamic instability in non-Newtonian fluids, when the theoretical homogeneous stress-strain-rate constitutive relation exhibits multi-valued behavior, as in theories of polymer melts 111,12] and worm-like micelles [13,14. Such models are often used to describe, for example, the spurt effect, whereby the flow rate of a fluid in a pipe changes discontinuously as a function of applied pressure drop [15]. The most important unresolved question about non-monotonic flow curves, such as those in Fig. 11a, is: what determines the stress, if any, at which the system phase separates into 'bands'? Suggestions have included (a) variational hypotheses (Ref. [16]); (b) assuming the stress at the top of the stable viscous branch is selected ("top jump") 12,13,17]; (c) including geometrical effects [18]; and (d) incorporating (physically present) non-local contributions to the stress 88.20$]$. In this Letter we pursue (d) and explore in detail the utility of constitutive inhomogeneous effects in resolving the issue of stress selection in complex fluids, which has occupied the rheology and physics communities in the guise of either unstable flows [19] or non-equilibrium phase transitions [8].

After a general discussion we introduce semi-phenomenological equations of motion for rod-like molecules in solution, extending the Doi model [21] to inhomogeneous flows. Our study has the following goals and results: (1) We present a general recipe for computing phase separation under flow, and hence the experimentally measured rheological behavior; (2) We point out that the proper field variable (either stress or strain rate) may not be unique, a feature absent from equilibrium systems; (3) Using concepts developed for dynamical systems theory we conclude that stress selection of models with nonlocal (in space) differential constitutive relations in planar shear flow is unique; i.e., as with equilibrium phase transitions, it occurs along a hyper-surface of lower di- mension than that of the field variable space. Our discussion is facilitated by examining the stress-strain-ratecomposition surface, a representation we have not seen before and hope will become commonplace.
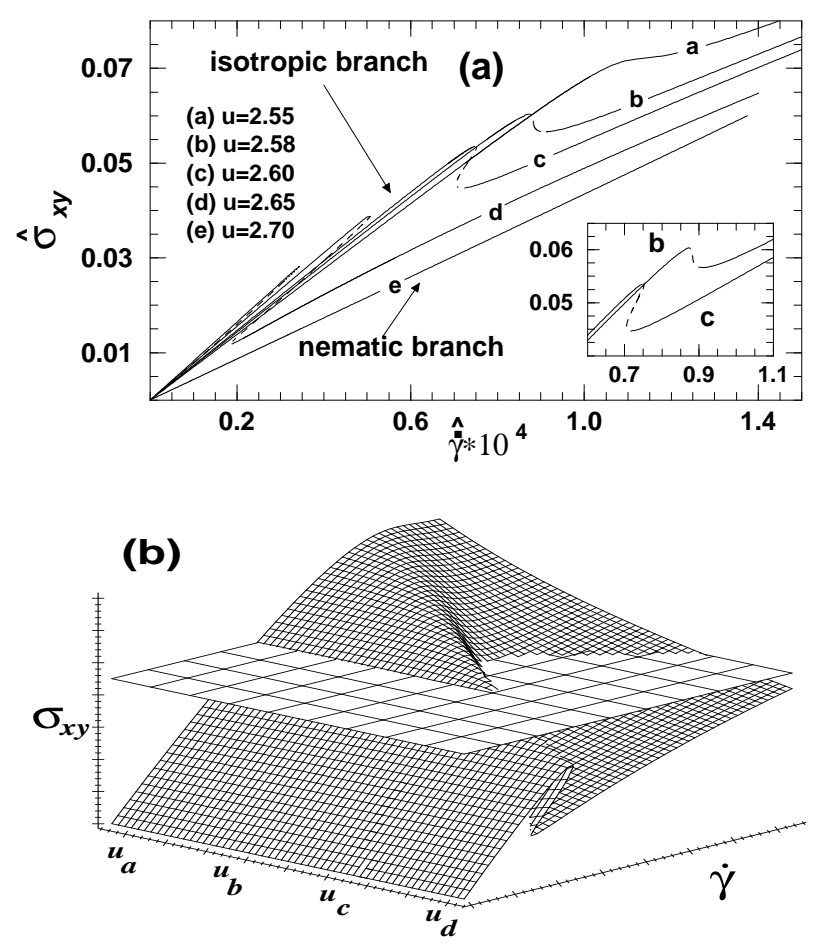

FIG. 1. (a) Homogeneous stress $\hat{\sigma}_{x y}$ vs. strain rate $\widehat{\dot{\gamma}}$ behavior for various excluded volumes $\left(u \equiv \phi L \nu_{2} / \alpha\right)$ as calculated from the Doi model with quadratic closure [21,23]. We use the dimensionless parameters $\widehat{\dot{\gamma}} \equiv \dot{\gamma} /\left(6 D_{\text {ro }} \nu_{1} \nu_{2}^{2}\right)$ and $\hat{\sigma}_{x y} \equiv \sigma_{x y} \nu_{2} \log L /\left(9 \pi \eta_{s} D_{r o}\right)$. Dotted lines mark unstable branches. The plane in (b) is at fixed stress. At $u_{e}$ both isotropic and nematic phases are metastable in zero shear. Here and below $L=5$.

The curves in Fig. 17a are reminiscent of pressurevolume isotherms in a liquid-gas system and, accordingly, we seek to construct a 'phase diagram' by pursuing an analogy between homogeneous stable steady 
states and equilibrium phases. As in equilibrium, nonequilibrium 'phases' may be separated, in field variable space, by hyper-surfaces representing continuous (e.g. critical points) or discontinuous ('first-order') transitions [8,1]. Coexistence implies an inhomogeneous state spanning separate branches of the homogeneous flow curves. In contrast to equilibrium bulk phases, where field variables are uniquely identified, here we may consider phase coexistence at a given shear stress, with the interface parallel to the vorticity-velocity plane (curves $b-e$ ); or a given strain rate (curves $c-e$ ), with the interface parallel to the velocity-velocity gradient plane. The appropriate field variable is thus determined by the nature of the constitutive relation (for curve $b$, common strain rate is impossible), or even by the flow history or the rheometer (e.g. fixing the stress or strain rate in curves $c-e$ ). In this Letter we compute coexistence at a common stress, but note that computation for a common strain rate is analogous. In equilibrium, minimizing the total free energy leads to equality of field variables between two phases and the Maxwell common tangent condition (e.g. the equal osmotic pressure condition, aided by equal chemical potential, in rod suspensions 22]). In dynamics, the former follows from the equations of motion plus the stationary condition, with an extra shear degree of freedom. However, the lack of a criterion to replace the latter leaves an unresolved degeneracy (e.g. which stress is selected for a given averaged strain rate). Flow experiments on worm-like micelles find a well-defined transition stress for the onset of banded flows [2,3,5,6, which suggests that the degeneracy is not physical.

For the thermotropic (i.e. restricted to the melt composition) I-N transition under flow, Olmsted and Goldbart [8] resolved this degeneracy by rejecting those possibilities which did not admit a stationary interface solution and found, numerically, an apparently uniquely selected state. They included gradient terms, which penalize (energetically) spatial microstructure variations and dictate the interface structure. The importance of gradient terms was also recognized in Refs. [23, 19,20. The existence condition of a stationary interface selects one among a band of possible coexisting solutions, and is fundamentally different from augmenting a local constitutive model with a variational principle [16 or assuming selection at the limits of stability [12, 13, 17]. Below we justify the uniqueness of a selected state.

Now we proceed with our calculation. The free energy of a solution of rod-like molecules of volume fraction $\phi$ in an athermal solvent (e.g. as in Ref. 22]) is given by

$$
\begin{aligned}
\mathcal{F}(\phi, \boldsymbol{Q}) & =k_{B} T \int d^{3} r\left\{\frac{\phi}{v_{r}} \log \phi+\frac{(1-\phi)}{v_{s}} \log (1-\phi)\right. \\
& +\frac{\phi}{v_{r}}\left[\frac{1}{2}\left(1-\frac{1}{3} u\right) \operatorname{Tr} \boldsymbol{Q}^{2}-\frac{1}{3} u \operatorname{Tr} \boldsymbol{Q}^{3}+\frac{1}{4} u\left(\operatorname{Tr} \boldsymbol{Q}^{2}\right)^{2}\right. \\
& \left.\left.+\frac{1}{2} K\left(\nabla_{\alpha} Q_{\beta \lambda}\right)^{2}\right]+\frac{1}{2} g(\nabla \phi)^{2}\right\}
\end{aligned}
$$

Here $v_{r}$ and $v_{s}$ are rod and solvent monomer volumes;
$Q_{\alpha \beta}$ is the nematic order parameter tensor 25]; and $u \equiv \phi L \nu_{2} / \alpha$ is Doi's excluded volume parameter [21], where $L$ is the rod aspect ratio and $\nu_{2}$ and $\alpha$ are $\mathcal{O}(1)$ geometrical prefactors. This free energy includes the entropy of mixing, the orientational contribution yielding an I-N transition [21, and spatial correlations of composition and nematic order within the one constant approximation. The phenomenological gradient terms may, in principle, be calculated from a microscopic model.

The equations of motion are [21,24,26]:

$$
\begin{aligned}
\rho\left(\partial_{t}+\mathbf{v} \cdot \boldsymbol{\nabla}\right) \mathbf{v} & =\boldsymbol{\nabla} \cdot \boldsymbol{\sigma}(\phi, \boldsymbol{\kappa}, \boldsymbol{Q}) \\
\left(\partial_{t}+\mathbf{v} \cdot \boldsymbol{\nabla}\right) \boldsymbol{Q} & =\boldsymbol{F}(\boldsymbol{\kappa}, \boldsymbol{Q})+\boldsymbol{G}(\phi, \boldsymbol{Q}) \\
\left(\partial_{t}+\mathbf{v} \cdot \boldsymbol{\nabla}\right) \phi & =-\nabla \cdot \mathbf{J}
\end{aligned}
$$

with $\kappa_{\alpha \beta}=\nabla_{\beta} v_{\alpha}$ and $\rho$ the density. The stress tensor is

$$
\boldsymbol{\sigma}=-p \mathbf{I}+2 \eta \boldsymbol{\kappa}^{s}+\boldsymbol{\sigma}_{r e v}(\phi, \boldsymbol{Q}),
$$

where $\kappa_{\alpha \beta}^{s} \equiv\left(\kappa_{\alpha \beta}+\kappa_{\beta \alpha}\right) / 2$ and we take $\eta$ to be the solvent viscosity $\eta_{s}$ for simplicity. The reversible stress due to the nematic order is [21, 8,24

$$
\boldsymbol{\sigma}_{r e v}=-3 \boldsymbol{H}+\boldsymbol{H} \cdot \boldsymbol{Q}-\boldsymbol{Q} \cdot \boldsymbol{H}-\nabla Q_{\alpha \beta} \frac{\delta \mathcal{F}}{\delta \nabla Q_{\alpha \beta}},
$$

where $\boldsymbol{H}=-\delta \mathcal{F} / \delta \boldsymbol{Q}$. The isotropic stress from the force density $(\nabla \phi) \delta \mathcal{F} / \delta \phi$ [26] has been neglected. In Eq. (3) the (reactive) ordering term $\boldsymbol{F}$ is given by

$$
\boldsymbol{F}(\boldsymbol{\kappa}, \boldsymbol{Q})=\frac{2}{3} \boldsymbol{\kappa}^{s}+\boldsymbol{\kappa} \cdot \boldsymbol{Q}+\boldsymbol{Q} \cdot \boldsymbol{\kappa}^{T}-2\left(\boldsymbol{Q}+\frac{1}{3} \boldsymbol{I}\right) \operatorname{Tr}(\boldsymbol{Q} \cdot \boldsymbol{\kappa}) .
$$

For simplicity, we have chosen the form appropriate for an infinite aspect ratio 21]. The dissipative portion $\boldsymbol{G}$ is

$$
\boldsymbol{G}(\phi, \boldsymbol{Q})=\frac{6 \nu_{1} D_{r \mathrm{o}} v_{r}^{3}}{k_{B} T \phi^{3} \ell^{6}\left(1-\frac{3}{2} \operatorname{Tr} \boldsymbol{Q}^{2}\right)^{2}} \boldsymbol{H},
$$

where $D_{r_{0}}$ is the single-rod rotational diffusion coefficient, $\nu_{1}$ is a geometric prefactor, and $\ell$ is the rod length [21]. The chemical potential $\mu$ drives the current $\mathbf{J}$,

$$
\mathbf{J}=-\boldsymbol{M} \cdot \nabla \mu
$$

where $\boldsymbol{M}$ is the mobility tensor and $\mu \equiv \delta \mathcal{F} / \delta \phi$.

For other systems, equations like Eqs. (4) and (3) govern the conserved and broken-symmetry (or other longlived) variables, respectively. For some local models, internal dynamics (Eq. 3) can be eliminated to give the stress as a history integral over the strain rate. In polymer melts [1], and worm-like micelles 14 far from a nematic regime, this leads to non-monotonic stress-strainrate curves.

We seek stable steady-state solutions to Eqs. (2-14) for planar shear, $\mathbf{v}(\mathbf{r})=\dot{\gamma} y \hat{\mathbf{x}}$. Integrating Eqs. (2, 4) along $y$ yields $\sigma_{x y}(\phi, \dot{\gamma}, \boldsymbol{Q})=\sigma_{0}$ and $\mu(\phi, \boldsymbol{Q})=\mu_{0}$, where $\sigma_{0}$ is the applied stress. One integration constant of Eq. (4) is zero from the boundary condition $J_{y}=0$, while $\mu_{0}$ is determined below [27]. For homogeneous solutions, $\boldsymbol{Q}$ 
may be eliminated from $\sigma_{x y}$ and $\mu$ using Eq. (3). The stress is shown in Fig. 11. Because $\mathcal{F}(\phi, \boldsymbol{Q})$ describes an I-N transition, multiple roots for $\boldsymbol{Q}$ may exist at a given stress [8,9], with distinct strain rates.

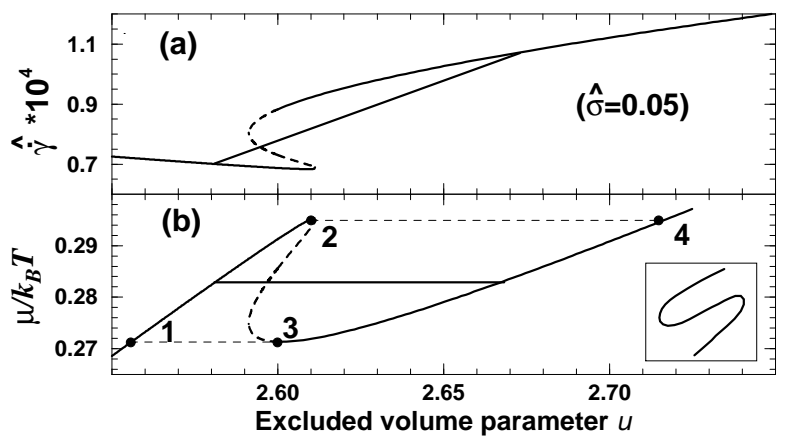

FIG. 2. (a) Reduced strain rate $\widehat{\dot{\gamma}}(u)$ for the stress contour in Fig. 1 b b. (b) $\mu(u)$ along the curve in (a). A selected pair has been indicated in (a) and (b). The inset shows another (topologically) possible $\mu(u)$. Dashed lines mark the limits of stability of coexisting pairs.

For coexistence at fixed stress $\sigma_{0}$ (the interface has a normal parallel to $\hat{y}$ ), a stress contour traces a line in the $(\dot{\gamma}-\phi)$ plane (Fig 2a). We plot $\mu(\phi)$ along this line in Fig. 2b. Coexisting phases must have the same $\mu$. For concentrations $\bar{\phi}$ outside points 1 and $4, \mu_{0}$ is determined uniquely. Between these points, we must determine $\mu_{0}$ at which two phases coexist. Following Ref. [8], we select the $\mu_{0}$ which permits a stationary inhomogeneous solution satisfying $\sigma_{x y}=\sigma_{0}, \mu=\mu_{0}$ and Eq. (3) $(0=\boldsymbol{F}+\boldsymbol{G})$. This is not an auxiliary assumption, but follows from the inhomogeneous equations of motion. Given a selected $\mu_{0}$ and a mean concentration $\bar{\phi}$, the portions of the two phases are fixed by the lever rule, and the mean shear rate can be calculated. In the static limit $(\dot{\gamma}=0)$ the stationary interface condition follows from minimizing $\mathcal{F}(\phi, \boldsymbol{Q})$ with a given $\bar{\phi}$ constraint. Functional minimization with respect to $\phi(y)$ allows the interface position to move, thereby adjusting the ratio of the two phases and recovering the common tangent construction.

To see how $\mu_{0}$ can be selected uniquely, note that the stationary solutions to Eq.(3), $\sigma_{x y}=\sigma_{0}$, and $\mu=\mu_{0}$, are nonlinear ordinary differential equations (ODE) involving $\partial / \partial y$. These ODE may be converted into an equivalent set of first order ODE with dependent variables $\{\phi, d \phi / d y, \dot{\gamma}, \boldsymbol{Q}, d \boldsymbol{Q} / d y\}$. In the ODE phase space, the interfacial solution corresponds to a trajectory (a 'heteroclinic saddle connection') joining two fixed points (the homogeneous states). As $\mu_{0}$ changes for fixed $\sigma_{0}$, the phase flow changes catastrophically at those isolated values of $\mu_{0}$ where the desired trajectory exists. [This is proven for differential constitutive relations in planar flow [24] by showing that the saddle connection, assuming it exists, is of the non-transverse type [28]]. This explains the apparent uniqueness of a selected $\mu_{0}$, given $\sigma_{0}$, found numerically. Our (one-dimensional) solution sup- ports the existence of such a solution in the modified Doi model, and is stable against perturbations in $y$. Because non-local effects (see Ref. [23, 19] for diffusion effects) always exist in reality, and pathological degeneracies only occur in local models, we expect that models that can resolve the interface structure have unambiguous phase diagrams.

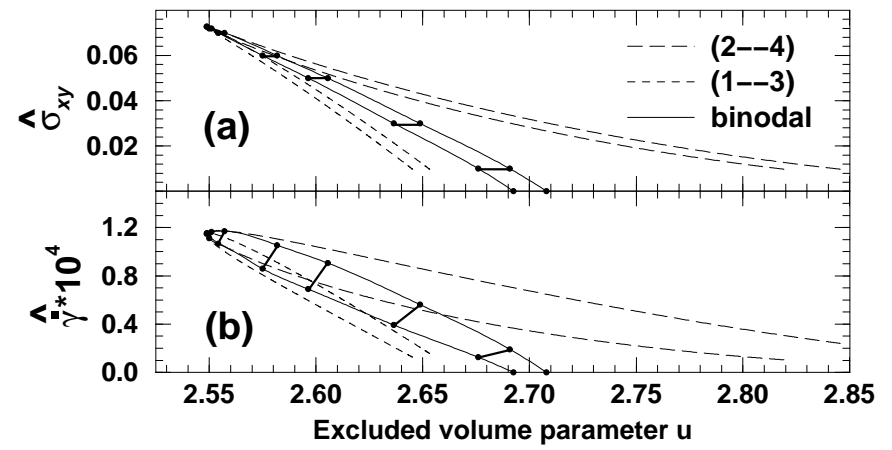

FIG. 3. Phase diagram in the $\left(\hat{\sigma}_{x y}-u\right)(\mathrm{a})$ and $(\hat{\dot{\gamma}}-u)(\mathrm{b})$ planes. Broken lines trace the loci of points $1-4$ in Fig. $\mathrm{A}$, and the solid lines are calculated tie lines.

The selection criterion determines the tie lines on Fig. 2, and varying the stress yields the phase diagram of Fig. 3b 24]. In the latter, for $\dot{\gamma}=0$ the tie line is horizontal, and for $\dot{\gamma}>0$ tie lines have positive slope, because the more concentrated nematic phase has a lower effective viscosity. For models where $\mu(\phi)$ has the shape of the inset of Fig. 2 $b$ b, tie lines have negative slopes.



FIG. 4. $\hat{\sigma}_{x y}$ vs. $\hat{\overline{\dot{\gamma}}}$ for various compositions. To read this from Fig. 3, choose a $u$ and increase the stress. In the two-phase region jump from stress tie line to stress tie line until the one phase region is reached.

Fig. 1 shows the stress-averaged-strain-rate curves as would be measured in an experiment. Here the coexistence region comprises lines with positive slopes. For models with vertical tie lines in Fig. 3 $\mathrm{b}$, indicating a composition-independent transition (as in the DoiEdwards model of polymer melts), the plateau would be flat. This is a graphical expression of the explanation of a sloped plateau given by Schmitt et al. [17] (however, they assumed "top-jumping"). In general one must vary both average concentration and strain rate to maintain constant shear stress, a situation familiar from equilibrium multi-component phase coexistence. The 'plateau' need not be a straight line; its shape is determined by the change in splay of the tie lines with increasing strain rate 
in Fig. [3b. Experiments on wormlike micelles [2] have found the plateaus to be steady states and the 'spines' to be metastable branches.

In summary, the steps to compute phase separation under flow are: (1) Determine the full inhomogeneous equations of motion; (2) Analyze stable homogeneous flows. (3) Choose the candidate field variable for phase coexistence. Multiple choices (e.g. stress or strain rate, according to interface geometry) must be analyzed separately. (4) Identify pairs of phases with the same field variables, as in Fig. I b and Fig. 2 $2 \mathrm{~b}$ for $\sigma_{x y}$ and $\mu$. (5) Determine the phase diagram (in field variables) by requiring a stationary interface between homogeneous solutions. To compare with experiments, density variables can be used, as in Fig. 3, to find (6) the tie lines where the lever rule applies and (7) the space-averaged stress-strain-rate relations (Fig. (4)). Other multi-component systems retain the structure of Eqs. (2 2 ), with Eq. (3) governing the dynamics of some structural order parameter.

We have calculated coexistence at fixed stress, while curves $c-e$ in Fig. 17a admit, in principle, coexistence at fixed strain rate. A full solution of Eqs. (2-1) requires analyzing both interface directions, which is reminiscent of, and may be relevant to, the problem of the orientation of diblock lamellar phases in shear [30]. Also, we have only considered flow-aligning solutions to the Doi model. Another, so-called 'log-rolling', solution is stable at high shear rates [29] and will be included in a complete treatment [24]. The extended Doi model is appropriate for hard rod suspensions [22], and we welcome experiments on these systems. The flow instability is due to perturbation of an equilibrium phase transition; while systems such as worm-like micelles probably possess some combination of perturbed (I-N) and dynamic transitions [5, 14], which could yield a stress-strain-rate-composition surface (Fig. 1]b) with multiple folds.

We have not considered the important problem of the stability of an undulating interface, which may restrict the choice of field variables [30]. Stability analysis by Renardy 31 on banded flow in the Johnson-Segelmann model (which has the qualitative behavior of curve $b$ in Fig. 1 1a, but no gradient terms) revealed a stationary interface at any stress in the two-stress region, and a family of unstable high-wavenumber undulations. Gradient terms break the stress degeneracy $[19,20,24$ and introduce a stabilizing surface tension and dissipation within the interface as it moves, which affects the stability analysis. Finally, complementary to the planar shear case analyzed above, Greco and Ball [18], have recently demonstrated the important result that, for a JohnsonSegelmann fluid in cylindrical Couette flow, a stationary interface exists at a selected stress: coexistence is apparently influenced by the curved boundary geometry of the flow.

We thank J.-F. Berret, M. Cates, F. Greco, P. Goldbart, J. Harden, R. Larson, G. Leal, T. McLeish, G. Porte, and N. Spenley for many discussions; the Isaac Newton Institute, where this work was begun; and (C.-
Y. D. L.) the Colloid Technology Programme for support.

* $\quad$ e-mail: phy6pdo@irc.leeds.ac.uk

$\dagger \quad$ e-mail: cydl1@phy.cam.ac.uk

[1] C. R. Safinya, E. B. Sirota, and R. J. Plano, Phys. Rev. Lett. 66, 1986 (1991).

[2] J.-F. Berret, el al., Europhys. Lett. 25, 521 (1994); J.-F. Berret, D. C. Roux, and G. Porte, J. Phys. II (France) 4, 1261 (1994).

[3] H. Rehage and H. Hoffmann, Mol. Phys. 74, 933 (1991).

[4] D. Roux, F. Nallet, and O. Diat, Europhys. Lett. 24, 53 (1993).

[5] V. Schmitt, et al., Langmuir 10, 955 (1994).

[6] P. T. Callaghan, et al., J. Phys. II (France) 6, 375 (1996).

[7] S. Hess, Z. Naturforsch. 31a, 1507 (1976).

[8] P. D. Olmsted and P. M. Goldbart, Phys. Rev. A41, 4578 (1990); ibid, A46, 4966 (1992).

[9] H. See, M. Doi, and R. Larson, J. Chem. Phys. 92, 792 (1990).

[10] M. E. Cates and S. T. Milner, Phys. Rev. Lett. 62, 1856 (1989).

[11] M. Doi and S. F. Edwards, The Theory of Polymer Dynamics (Clarendon, Oxford, 1989).

[12] M. E. Cates, T. C. B. McLeish, and G. Marrucci, Europhys. Lett. 21, 451 (1993).

[13] N. A. Spenley, M. E. Cates and T. C. B. McLeish, Phys. Rev. Lett. 71, 939 (1993).

[14] M. E. Cates, J. Phys. Chem. 94, 371 (1990).

[15] T. C. B. McLeish and R. C. Ball, J. Poly. Sci. B-Poly. Phys. 24, 1735 (1986).

[16] G. Porte, J.-F. Berret, and J. L. Harden, unpublished (1996).

[17] V. Schmitt, C. M. Marques, and F. Lequeux, Phys. Rev. E52, 4009 (1995).

[18] F. Greco and R. C. Ball, to be published (1996).

[19] J. R. A. Pearson, J. Rheol. 38, 309 (1994).

[20] N. A. Spenley, X. F. Yuan, and M. E. Cates, J. Phys. II (France) 6, 551 (1996).

[21] M. Doi, J. Poly. Sci: Poly. Phys. 19, 229 (1981); N. Kuzuu and M. Doi, J. Phys. Soc. Jap. 52, 3486 (1983).

[22] P. A. Buinin and H. N. W. Lekkerkerker, J. Phys. Chem. 97, 11510 (1993).

[23] A. W. El-Kareh and L. G. Leal, J. Non-Newt. Fl. Mech. 33, 257 (1989).

[24] P. D. Olmsted and C.-Y. D. Lu, to be published.

[25] P. G. de Gennes and J. Prost, The Physics of Liquid Crystals, 2nd ed. (Clarendon, Oxford, 1993).

[26] P. C. Hohenberg and B. I. Halperin, Rev. Mod. Phys. 49, 435 (1977).

[27] In the few cases where the final phase diagram of $\sigma_{x y}$ and $\mu$ has a transition line parallel to the $\mu$ axis, one must first fix $\mu_{0}$, and then determine $\sigma_{0}$.

[28] See, e.g., R. H. Abraham and C. D. Shaw, Dynamics The Geometry of Behavior, Part 3 (Aerial Press, Santa Cruz, 1985), p.53. 
[29] A. V. Bhave, et al., J. Rheol. 37, 413 (1993).

[30] M. Goulian and S. T. Milner, Phys. Rev. Lett. 74, 1775
(1995).

[31] Y. Y. Renardy, Th. Comp. Fl. Mech. 7, 463 (1995). 